\title{
Integração da interface phantom ao sistema tutor inteligente para o ambiente de simulação médica
}

\author{
Jairo Simão Santana Melo*, Lourdes Mattos Brasil, Carlos Eduardo Balbuena Panerai, \\ Ana Paula Bernardi da Silva
}

Resumo O presente trabalho aborda e analisa o uso da Realidade Virtual (RV) em Sistemas Tutores Inteligentes (STI), buscando evidenciar a cooperação entre as duas áreas. Neste sentido, as teorias de Inteligência Artificial (IA) contribuíram para a estruturação de ambientes interativos de ensino com as melhores práticas de STI e RV, entre elas: facilidade de acesso, individualização do ensino, conteúdo lapidado, modelado e selecionado, um ambiente imersivo, interativo, intuitivo, destacando a integração do robô Phantom ao ambiente 3D e o seu relacionamento com a ontologia do projeto. Um estudo de caso foi realizado na área de anatomia ósseocraniana para cursos de graduação na área da saúde. O processo de validação do protótipo e da arquitetura proposta teve apoio de professores e um grupo de 120 estudantes da área da saúde da UCB e da UnB. Como os cursos eram de características distintas, dividiu-se a validação em dois grupos com propósitos qualificados. O grupo A ficou responsável pela validação da arquitetura/tecnologia formado pelos estudantes de medicina do $1^{\circ}$ semestre da disciplina de anatomia de ambas as instituições. $\mathrm{O}$ grupo B foi utilizado para a validação do conceito/conteúdo e formado por estudantes de enfermagem e biomedicina também do $1^{\circ}$ semestre da disciplina de anatomia, sendo que esses estavam sob a responsabilidade do mesmo professor e tiveram a mesma forma de avaliação. Os estudantes de biomedicina tiveram acesso ao sistema, enquanto que os de enfermagem tiveram apenas o ensino convencional, sem acesso ao ASM. O objetivo foi verificar se a utilização do ASM como foi implementado contribuiu para o ensino da anatomia humana. O processo de aprendizado foi alocado no trabalho como uma possibilidade de expansão da arquitetura e evolução desse trabalho, acrescentando uma rede neural artificial entre a interação do estudante e o tipo de conteúdo a ser solicitado da ontologia. Portanto, através de um questionário on-line realizado com o apoio de um especialista da área de saúde, o ASM teve resultados satisfatórios.

Palavras-chave Ontologia, Sistema tutor inteligente, Interfaces hápticas, Realidade virtual.

\section{Integration of the phantom interface on intelligent tutorial system for the medical simulation environment}

\begin{abstract}
The present study describes an analysis of the use of Virtual Reality (VR) in Intelligent Tutorial Systems (ITS), aiming to show the cooperation between the two areas. Consequently, Artificial Intelligence (AI) theories contributed to the structuring of interactive teaching environments with the best ITS and VR practices, among these: easy access, individualized teaching, refined, modeled and selected content, an intuitive, immersive, interactive environment, particularly the integration of the Phantom robot in a $3 D$ environment and its relationship to the ontology of the project. A case study was completed for undergraduates majoring in health, in the area of cranial-skeletal anatomy. The prototype and proposed architecture validation process was supported by professors and a group of 120 students from UCB and UnB in the area of health. As the majors had distinct characteristics, the validation was divided into two groups with qualified proposals. Group A was responsible for the validation of the architecture/technology and consisted of the medical students in their $1^{\text {st }}$ semester of anatomy from both of the institutions. Group B was used for the validation of concept/content and consisted of students in nursing, and biomedicine, also in their $1^{\text {st }}$ semester of anatomy, under the supervision of the same professor, and undergoing the same evaluation process. The students in biomedicine had access to the system, while the nursing students had conventional teaching methods, without access to the Medical Simulation Platform (MSP). The objective was to verify if the use of the MSP, as it was implemented, contributed to the teaching of human anatomy. The learning process was allocated in the work as a possibility of expansion of the architecture and evolution of this work, adding an artificial neural network between the interaction of the student and the type of content to be solicited from the ontology. Through an on-line questionnaire, administered with the help of a health expert, the MSP presented satisfactory results.
\end{abstract}

Keywords Ontology, Intelligent tutoring systems, Haptic devices, Virtual reality. 


\section{Extended Abstract}

\section{Introduction}

Evidence of the demand for innovation in teaching in the area of medicine is demonstrated by the increasing interest in Intelligent Tutorial Systems (ITS) (Bittencourt, 2006; Brasil, 2008) dedicated to a wide variety of areas in health, such as anatomy, physiology, pathology and surgical procedure simulation.

Accordingly, the general objective of this paper is to investigate the association of ITS with Virtual Reality (VR) environments by designing an architecture that makes possible its integration, both in terms of technology and content, facilitating visualization, navigation and interaction with the structures incorporated in $3 D$ environment through the resources of computer graphics and haptic interfaces.

ITS are generally built with the intuition of emphasizing the relation between text and image in the learning sequence determined by a specialist and with the support of pedagogical agents (Bittencourt, 2006). Nevertheless, the semantics of learning defined and modeled by a specialist is not structured in a $3 D$ environment, which ends up being a mere visualizing tool for $3 D$ objects. The objective of this study is to investigate the integrations of the virtual environment and the same learning semantics defined by the ITS, to create a channel of communication and interaction between the ITS and the VR (Melo, 2007), using ontology.

The ontology allows for the formal organization of content using a single vocabulary. This article combines two ontologies to refine the relationship between $3 D$ objects and the descriptive information on anatomy. The first Foundational Model of Anatomy (FMA) was developed by the University of Washington and is designed for formalizing knowledge in anatomy. The second is relative to the domain of VR and is based on the architecture of the $3 D$ processing framework and haptics developed by the University of Stanford.

After the integration of the two ontologies, they were submitted to the inference machine RACER PRO (Haarslev et al., 2004), seeking to find evidence of the development of possible hypotheses about the relationships described and a better redistribution of the original classes.

The ontology, by centralizing knowledge, allows the MSP to make available functions of bidirectional content navigation. In other words, both the ITS and the VR have access to the different formalized domains, allowing the learner to choose the most convenient form of navigation.

Through the JSF library and ActiveX technology, made available by Microsoft ${ }^{\mathbb{}}$, it was possible to pass on the characteristics of the ITS to a 3D environment and vice-versa, in a parameterized way. The haptic resources integral to this framework allow tactile contact with the $3 D$ structures selected, allowing the student to explore sensations of dimensions, textures and physical properties with weight, rigidity and elasticity. In this sense, when the client solicits the visualization of a $3 D$ structure, as well as the haptic interface, the ontology configures the simulation according to the domain selected, being then passed on to the ActiveX component, which shows the object in $3 D$. In this respect, it is possible to highlight the management of the haptic interface during the simulation, having as a principle, the relationships existent between the classes and the properties passed on by the Web service of ontological processing developed by this study.

\section{Validation process}

The validation process of the architecture was conducted by professors in medicine, nursing and biomedicine from two Brazilian universities (UCB and UnB), using the content of cranial anatomy in an anatomy course. The research, and therefore the instrument of evaluation, was structured considering its classification as quantitative, in laboratory and methodology. The MSP was implemented in a Web environment and the validation was completed by the application of an electronic questionnaire given to the students in the Majors cited above, subdivided in two groups: medicine from the two institutions, and nursing with biomedicine. All of the students were supervised by the same professor.

The validation evaluated the relevance of the following topics:

- Usability;

- Content;

- Virtual Reality;

- Teaching Systems;

The variables of the answers were organized considering the Boolean interval (i.e. 0 to 1) and fuzzy logic, to better quantify the process of evaluation of the MSP.

\section{Results}

The questionnaire showed that: $i)$ the use of technology in the field of medicine was recommended by about $80 \%$ of those interviewed; ii) the use of VR and the manipulation of objects contributed to the comprehension of anatomical structures; iii) the use of tactile interfaces associated to the VR was recommended by approximately $67 \%$ of those interviewed; iv) the quality of the content was tested with $67 \%$ stating that the system has improved the learning of anatomy.

\section{Discussion and conclusion}

Some operational problems were found with respect to the stability of the multi-use system, different operational systems, storing and retrieval of files.

The architecture of the system described in this study was valuable in the areas of health and education, proposing an ITS that formalizes the content in the form of ontologies, allowing for the relationship between different domains of knowledge in health through the navigation by possible varied links and bidirectional VR and ontology, and allows for the use of tactile interfaces.

This architecture can be updated with the objective of finding an evaluation process for the learning process and the interaction with other structures of human anatomy that demand simulation of surface deformation, as for example, the manipulation of mammary tissue. 


\section{Introdução}

A informática aplicada à área de saúde vem ganhando destaque nos últimos anos. Ela vem contribuindo para o processo de tomada de decisão médica, auxiliando e sugerindo diagnósticos, bem como no aperfeiçoamento do ensino médico. No que diz respeito à tecnologia da informação disponível hoje para a área de saúde, é possível dar um salto de qualidade por meio de ambientes capazes de prover rapidamente dados selecionados, lapidados e modelados de forma a apoiar o processo decisório médico (Orgun e Vu, 2006).

A informática em saúde vem fazendo uso de novas ferramentas e tecnologias da computação, como é o caso da Inteligência Artificial (IA), Realidade Virtual (RV), Interfaces Hápticas, Multimídia, Hipermídia e Internet. Atualmente, diversas iniciativas buscam a associação dessas abordagens para se obter maior precisão das informações médicas manipuladas (Liu e Uang, 2007; Rönkkö et al., 2006; Wilson e D'cruz, 2006). Mais especificamente, a demanda da área médica pela inovação do ensino é evidenciada pelo crescente interesse por Sistemas Tutores Inteligentes (STI) (Bittencourt, 2006; Brasil, 2008) voltados a diversas áreas da saúde, tais como anatomia, fisiologia, patologia e simulação de procedimentos cirúrgicos, principalmente o uso de interfaces hápticas, como os robôs phantoms (Faeth et al., 2008; Melo, 2007; Santos et al., 2009). A anatomia humana é uma das disciplinas básicas no ensino das ciências da saúde, sendo frequente a necessidade de consulta a livros-texto, a atlas de anatomia e mesmo a manipulação de cadáveres. Estes fatos motivaram o desenvolvimento do STI aplicado ao Ambiente de Simulação Médica (ASM), apresentado a seguir.

O objetivo desse trabalho consiste em investigar a associação de STI com ambientes de RV através da concepção de uma arquitetura que viabilize esta integração, facilite a visualização, navegação e a interação com as estruturas inseridas no ambiente 3D através de recursos de computação gráfica e interfaces hápticas.

\section{Materiais e Métodos}

A maioria dos STI disponíveis hoje busca enfatizar a relação entre texto e imagem em sequência de aprendizado determinada por um especialista e com o apoio de agentes pedagógicos (Bittencourt, 2006). Com a possibilidade de interação com ambientes virtuais, o aprendiz ganha mais um artifício de estudo (Bowman et al., 2001). Contudo, a semântica de aprendizado definida e modelada por um especialista não é estruturada no ambiente 3D. Ou seja, o ambiente virtual passa a ser um mero visualizador de objetos 3D. O objetivo deste trabalho é investigar a agregação ao ambiente virtual da mesma semântica de aprendizados definida para os STI, de forma a criar um canal de comunicação e interação entre STI e RV (Melo, 2007), por exemplo, pelo compartilhamento da mesma base de conhecimento. Neste sentido, o sistema ASM busca inicialmente estreitar o relacionamento entre STI e RV, tirando proveito do conceito de ontologias (Bezivin e Lemesle, 1997). Tal conceito permite garantir uma forma de acesso ao conhecimento de maneira organizada, formal e com um vocabulário único. Possibilita também a navegação no conhecimento de forma hierárquica (conhecimentos dentro do domínio da anatomia) e lateral (conhecimentos em patologia e fisiologia), através dos relacionamentos produzidos entre as classes.

O conhecimento é obtido da ontologia através de comentários adicionados às classes, da definição das hierarquias e relacionamentos, do vocabulário associado à classe, dos axiomas representados e das restrições e domínios adicionados em cada classe.

A ontologia elaborada no âmbito deste trabalho teve como base a Foundational Model of Anatomy (FMA) (Dameron et al., 2005), ontologia desenvolvida pela Universidade de Washington e destinada à formalização do conhecimento da anatomia. O projeto FMA traz no seu conjunto de relacionamentos uma associação primária em relação ao domínio da visualização 3D. Entretanto, a ontologia aqui proposta aprimora o relacionamento entre os dois domínios. A ontologia relativa ao domínio RV teve por base a arquitetura do framework de processamento 3D e háptico desenvolvida pela Universidade de Stanford (Conti, 2010), cuja hierarquia é apresentada na Figura 1, na qual são apresentados os diferentes domínios da RV, destacando-se os pacotes mundo, objeto $3 \mathrm{D}$ e gráfico, que auxiliam no processamento do objeto 3D importado e disponibilizado na cena. As subclasses deste pacote possibilitam a configuração da luminosidade do ambiente, posição de câmera, manipulação da malha de triângulos e interação com a placa gráfica através da biblioteca opengl.

O pacote input é responsável pela integração de dispositivos ao ambiente virtual, assim como a interação do dispositivo com o objeto 3D através das subclasses de colisão. O pacote matemática auxilia, principalmente, com métodos para manipulação de matrizes. Como uma aplicação desse nível depende de um elevado nível de consumo de recursos de processamento, a arquitetura CHAI modela o ambiente virtual utilizando a tecnologia de threads, que atribui funções diferentes a cada subrotina da arquitetura. Essas subrotinas são classificadas como thread gráfica, 


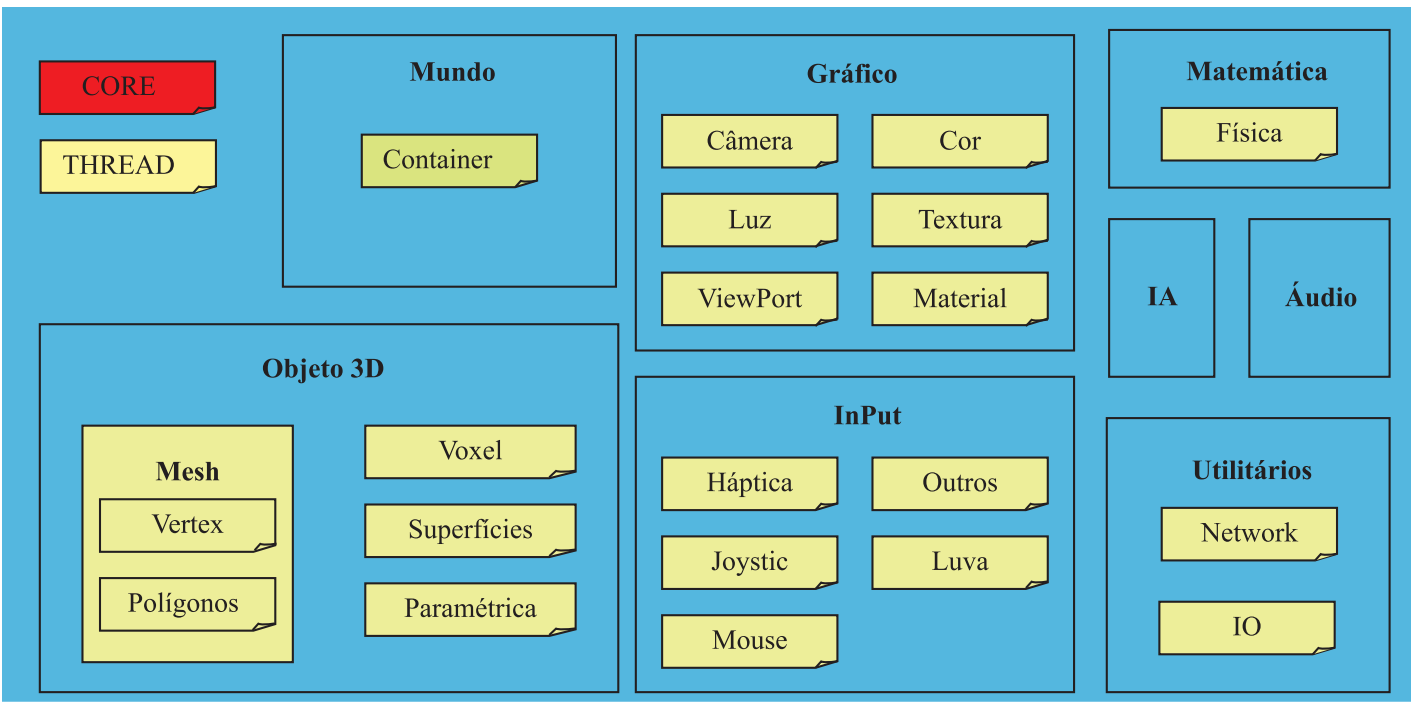

Figura 1. Arquitetura CHAI3D.

Figure 1. CHAI3D Architecture.

responsável pelo ambiente de redesenho gráfico, thread háptica, responsável pela interação com a interface háptica e a thread conteúdo, que manipula informação no ambiente $3 \mathrm{D}$, de forma que as três subrotinas também trocam informações através de memória compartilhada.

A ontologia foi desenvolvida no ambiente Protégé (Dameron et al., 2005), representando a hierarquia de classes, atributos, propriedades, restrições, axiomas e os relacionamentos internos descritos no CHAI3D. Essa ontologia, associada à FMA, possibilitou o casamento entre objetos 3D com informações descritivas da anatomia humana. É possível, assim, visualizar as classes descritas no FMA sobre diferentes focos, como pode ser visto na Figura 2.

Após a integração das duas ontologias, as mesmas foram submetidas à máquina de inferência RACER PRO (Haarslev et al., 2004), buscando evidenciar a geração de possíveis hipóteses sobre os relacionamentos descritos e uma melhor redistribuição das classes originais mostradas na Figura 1. Dentre as alterações propostas pela máquina de inferência, estão a incorporação da classe IA pela classe matemática devido à igualdade dos atributos, e a incorporação das classes Objeto 3D, Gráfico e Input como subclasse da classe Mundo em decorrência da associação de atributos. Nesse sentido, o ASM pode ser enquadrado como STI, pois gera conhecimento a partir de um conjunto de informações pré-processadas. Quanto às linhas de derivação de hipóteses, o ASM é classificado como de "encadeamento progressivo", onde a solução

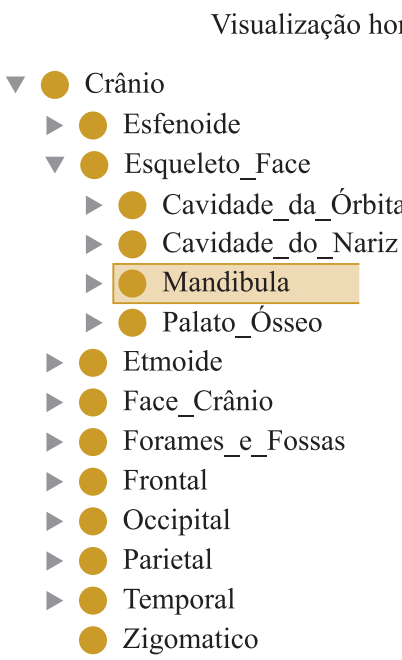

(i)

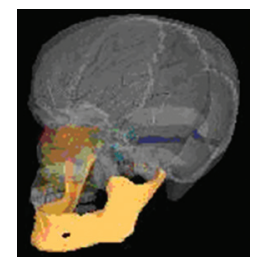

(ii)

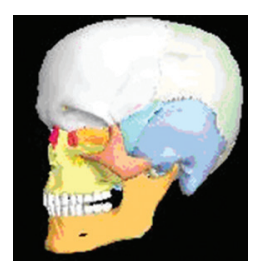

(iii)
Visualização vertical

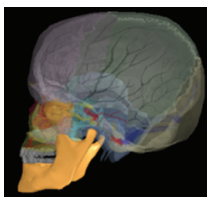

(iv)

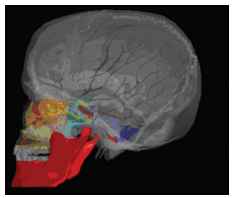

(v)

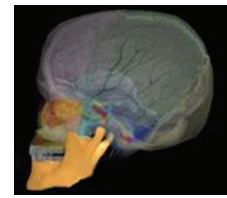

(vi)
Figura 2. i) Visualização hierárquica da ii) mandíbula em relação ao iii) crânio. Visualização lateral em relação aos domínios da iv) anatomia, v) patologia e vi) fisiologia.

Figure 2. i) Hierarchic view of ii) the mandible in relation to iii) the skull. View side in the fields of iv) anatomy, v) pathology and vi) physiology. 
de um problema tem como ponto de partida dados ou idéias (Russell e Norvig, 2004).

A integração de ontologias com ambientes de RV, como no ASM, contribui para as atuais técnicas de ensino, pois incorpora a possibilidade de cruzamento e navegação em diferentes domínios do conhecimento. O ASM proporciona um ensino reativo, isto é, depende da solicitação do aprendiz para a mudança de contexto. O contexto e a estrutura selecionada são visualizados em um ambiente 3D de acordo com as associações e atributos formalizados na ontologia, como pode ser observado na visualização do osso maxilar disposto na Figura 3. O maxilar é realçado dos demais ossos limítrofes a fim de se evidenciar a hierarquia de classes e os atributos de dimensão, posição e peso definidos no domínio Anatomia.

A ontologia, ao centralizar os conhecimentos, permite que o ASM disponibilize funcionalidades de navegação bidirecional de conteúdo. Ou seja, tanto o STI quanto a RV têm acesso aos diferentes domínios formalizados, permitindo que o aprendiz escolha a forma de navegação mais conveniente.

A interação bidirecional do ASM foi possível através da associação de tecnologias descritas na Figura 4, onde é predominante a presença da tecnologia Java no relacionamento com a ontologia, na modelagem dos serviços da camada de negócio encapsulada em um serviço web e na camada de apresentação do sistema. Entretanto, a interação com o ambiente virtual foi desenvolvida em $\mathrm{C}++$, pois as bibliotecas de processamento gráfico e interação háptica CHAI3D já estavam desenvolvidas nessa linguagem de programação com a da Universidade de Stanford.

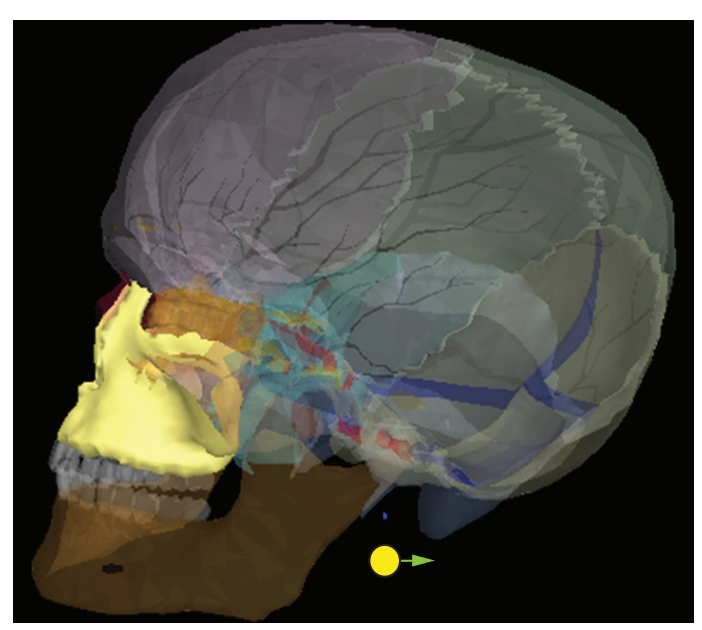

Figura 3. Visualização do osso maxilar sobre coordenação de ontologias.

Figure 3. Visualization of the maxilla bone under coordination of ontologies.
Nesse sentido, a maneira mais simples de integrar o ambiente 3D com o ASM foi através da tecnologia Active $X$, que possibilita adicionar uma janela de desenho gráfico do sistema operacional a uma página HTML. Para facilitar essa associação com a tecnologia Java, foi desenvolvida uma biblioteca do tipo TagLib em Java Server Faces (JSF) para exportar a geração do código HTML dentro do código Java, como é exemplificado na Figura 5.

Através dessa biblioteca JSF e da tecnologia ActiveX disponibilizada pela Microsoft ${ }^{\circledR}$ foi possível repassar a característica do STI para o ambiente 3D e vice-versa de forma parametrizada, como pode ser observado no exemplo anteriormente descrito onde são passadas as configurações do ambiente 3D tais como cor de fundo, dimensões de perspectiva, luminosidade e posição de câmera. A configuração do objeto 3D também é repassada pelo STI destacando-se o diretório de importação, objeto 3D a ser carregado, posição inicial do objeto em relação ao mundo, tipo de visualização em relação ao domínio de estudo (anatomia, patologia ou fisiologia) e informações de conteúdo definidas pelo especialista. Outros recursos, também repassados pelo STI ao ambiente 3D, são os parâmetros de dispositivos usados para visualização, interação e navegação do ambiente virtual, tais como óculos estereoscópicos e interfaces hápticas colocados à disposição desse projeto, destacando-se o braço robótico Phantom Omni (Faeth et al., 2008; Liu e Uang, 2007; Melo, 2007; Patel et al., 2006), que permite contato táctil com as estruturas 3D selecionadas possibilitando ao estudante sensações tácteis como dimensões, texturas e propriedades físicas como peso, rigidez, elasticidade, como é exemplificado na Figura 6.

Quando o cliente solicita a visualização de uma estrutura 3D, assim como o uso da interface háptica, a ontologia configura a simulação de acordo com o domínio selecionado, sendo em seguida repassada ao componente ActiveX que apresenta o objeto 3D. Nesse aspecto, é possível destacar o gerenciamento da interface háptica durante a simulação, tendo como princípio os relacionamentos existentes entre as classes e as propriedades repassadas pelo serviço web de processamento de ontologias desenvolvido por este trabalho. Tais propriedades são previamente configuradas por um especialista que determina a melhor forma de interação com as estruturas descritas na ontologia. Estes requisitos configuram o ambiente de $\mathrm{RV}$, sendo também repassados à interface de interação háptica quando ativada. Os parâmetros da simulação são atualizados na ontologia, com a finalidade de manter o estado da simulação. Este gerenciamento é exemplificado pelo diagrama esquemático representado na Figura 7. 


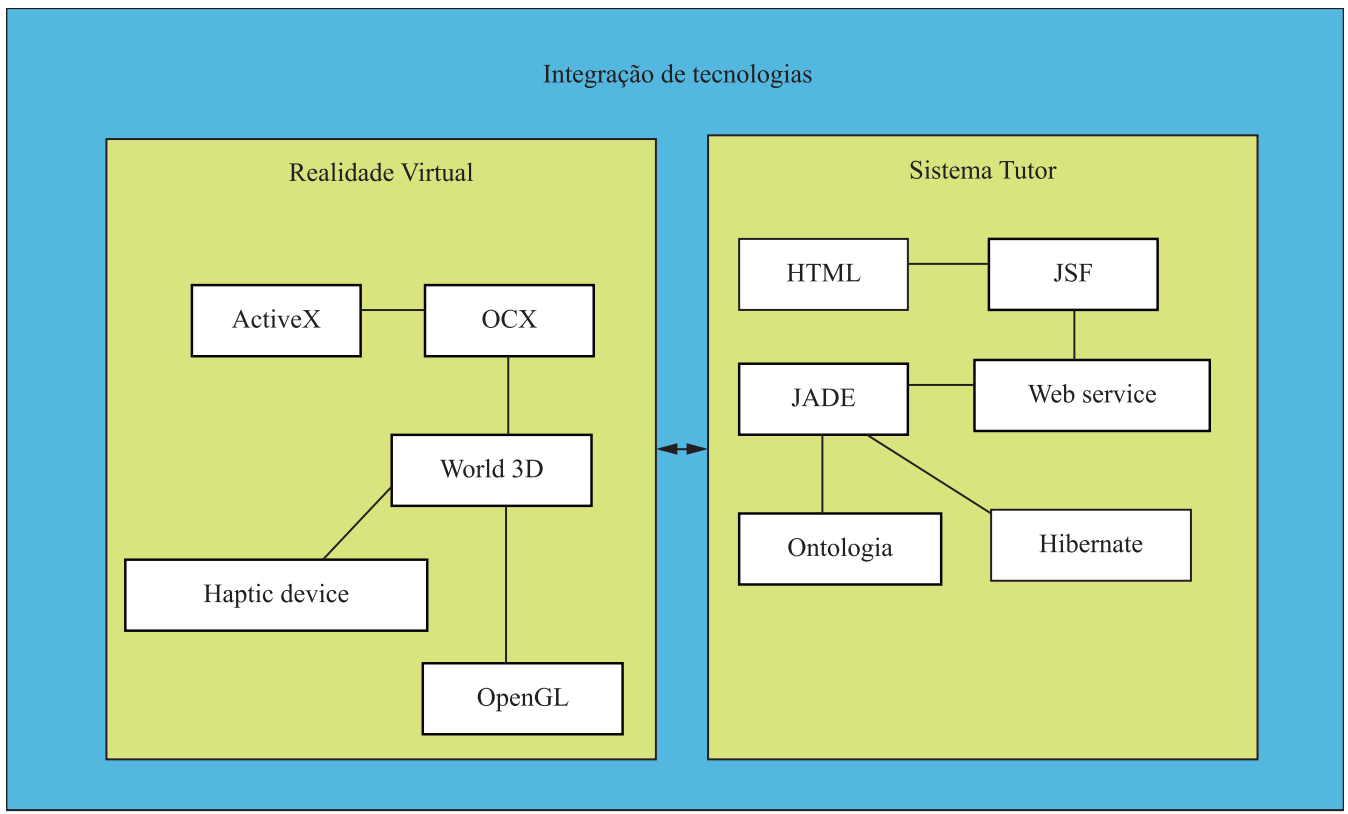

Figura 4. Arquitetura de integração e gerenciamento.

Figure 4. Architecture integration and management.

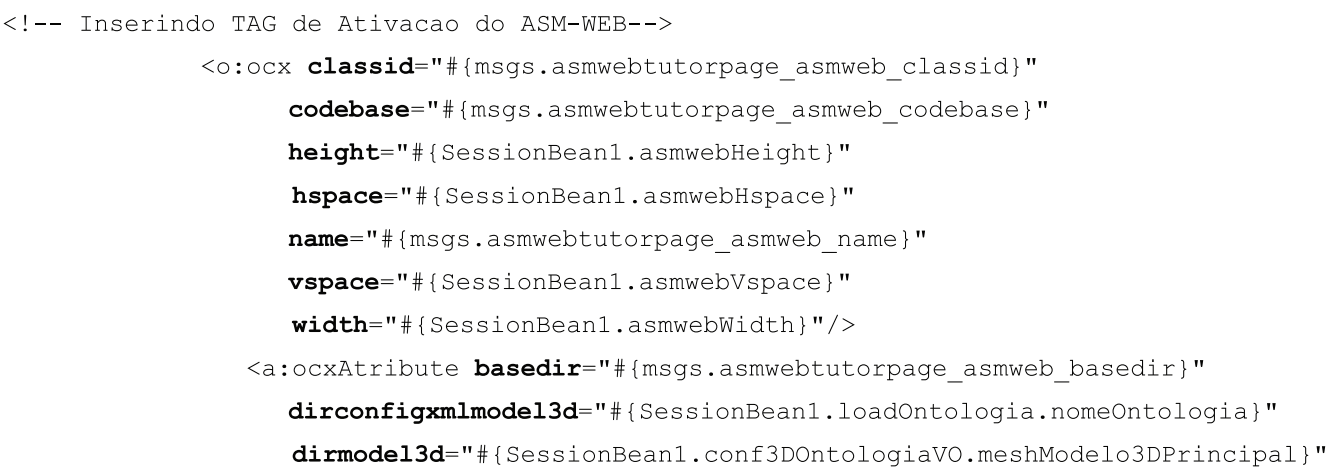

Figura 5. Tag Lib de integração Java com ActiveX.

Figure 5. Java Integration Tag Lib with ActiveX.

A integração entre os quatro conceitos base deste projeto, isto é, RV, interface háptica, STI e ontologia, proporcionariam um relacionamento pioneiro. Tendo como destaque o uso da ontologia como repositório central de disseminação de informação, possibilita que o especialista que não tem conhecimento do sistema atualize o conteúdo e os parâmetros utilizados na simulação sem a necessidade de um conhecimento técnico do sistema, ao contrário do modo convencional, onde o mesmo dependeria de uma instrução técnica para tal atividade.

\section{Processo de validação}

O processo de validação da arquitetura proposta foi realizado na área da anatomia ósseo-craniana e contou com o apoio de professores de informática, engenharia biomédica, especialista da área da saúde e um grupo de 120 estudantes da área da saúde de duas universidades brasileiras (UCB e UnB), como indicado na Tabela 1.

Por serem cursos com características distintas, dividiu-se a validação em dois grupos seguindo 
sugestão e necessidade do especialista: o grupo A, formado por estudantes de medicina da disciplina de anatomia do $1^{\circ}$ semestre de ambas as instituições, validou a arquitetura e a tecnologia; o grupo $\mathrm{B}$, formado por estudantes de enfermagem e biomedicina e também participantes da disciplina de anatomia do $1^{\circ}$ semestre, sob a responsabilidade do mesmo professor, validou os conceitos e o conteúdo. O objetivo foi verificar se a utilização do ASM como foi implementado contribuiu para o ensino da anatomia humana.

Do ponto de vista da abordagem do problema, esta é uma pesquisa quantitativa, porque visa diagnosticar a eficiência do uso de RV em STI destinados a aprendizes da área da saúde. Quanto aos meios, por se tratar de simulações feitas em computador, classifica-se como pesquisa de laboratório, porém com validações no domínio específico deste trabalho. Com relação à natureza e aos fins, este trabalho pode ser classificado como metodológico e aplicado (Moresi, 2003).

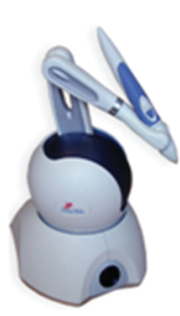

(i)

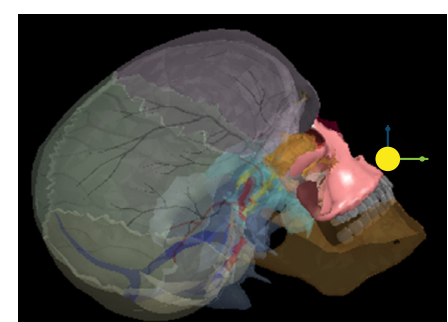

(ii)
Figura 6. i) Phantom Omni integrado ao ASM e ii) sua interação com o ambiente de RV.

Figure 6. i) Phantom Omni integrated to MSP and ii) its interaction with the VR platform.
Metodológico porque o estudo está associado a caminhos, formas, maneiras, procedimentos para atingir determinado fim; aplicado porque é, fundamentalmente, motivado pela necessidade de resolver problemas concretos, tendo, portanto, finalidade prática.

O ASM foi disponibilizado em ambiente web, representado na Figura 8 e a validação da arquitetura proposta foi realizada a partir da integração de um questionário eletrônico formulado junto com o especialista que foi disponibilizado ao estudante após finalizar o uso do sistema. O questionário continha grupos de perguntas relativos à usabilidade, conteúdo, RV e sistema de ensino, como pode ser observado na Tabela 2, com escopos de resposta que variaram desde sim e não até ruim, bom, razoável e ótimo. Com a experiência do especialista foi obtida uma faixa de valores para cada uma destas variáveis linguísticas compreendidas entre 0 e 1 , inclusive os extremos deste escopo foram utilizados nas respostas booleanas, em conformidade com a aplicação da teoria de lógica fuzzy (Brasil, 2008; Zadeh, 1965), de forma a quantificar melhor o processo de avaliação do ASM. Foi também objeto de observação o relacionamento da arquitetura ASM com os meios tradicionais de ensino,

Tabela 1. Distribuição de validação de dados.

Table 1. Distribution of the data validation.

\begin{tabular}{clcc}
\hline \multirow{2}{*}{ Grupo } & \multirow{2}{*}{ Curso } & \multicolumn{2}{c}{ Quantidade } \\
\cline { 3 - 4 } & & UCB & UnB \\
\hline A & Medicina & 30 & 30 \\
B & Enfermagem & - & 30 \\
& Biomedicina & - & 30 \\
\hline
\end{tabular}

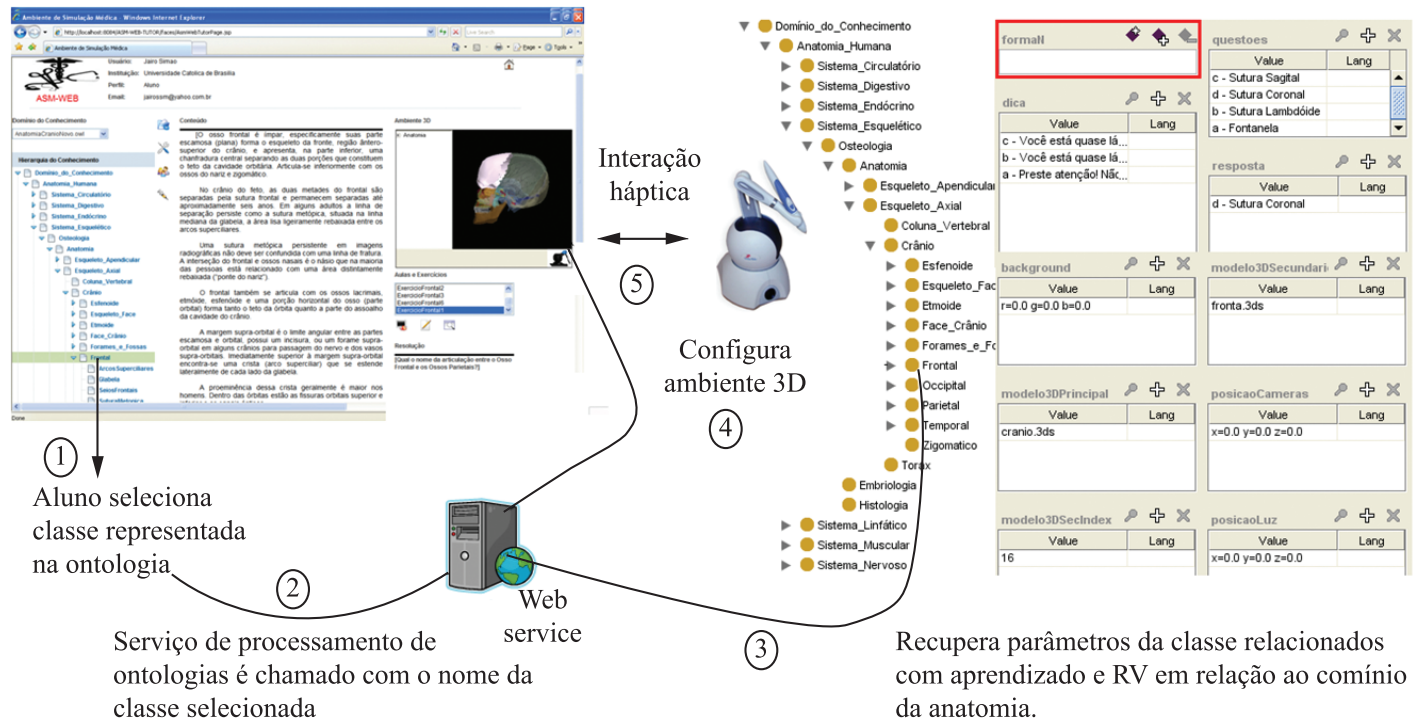

Figura 7. Exemplificação do gerenciamento da interface háptica.

Figure 7. Exemplification of haptics management interface. 


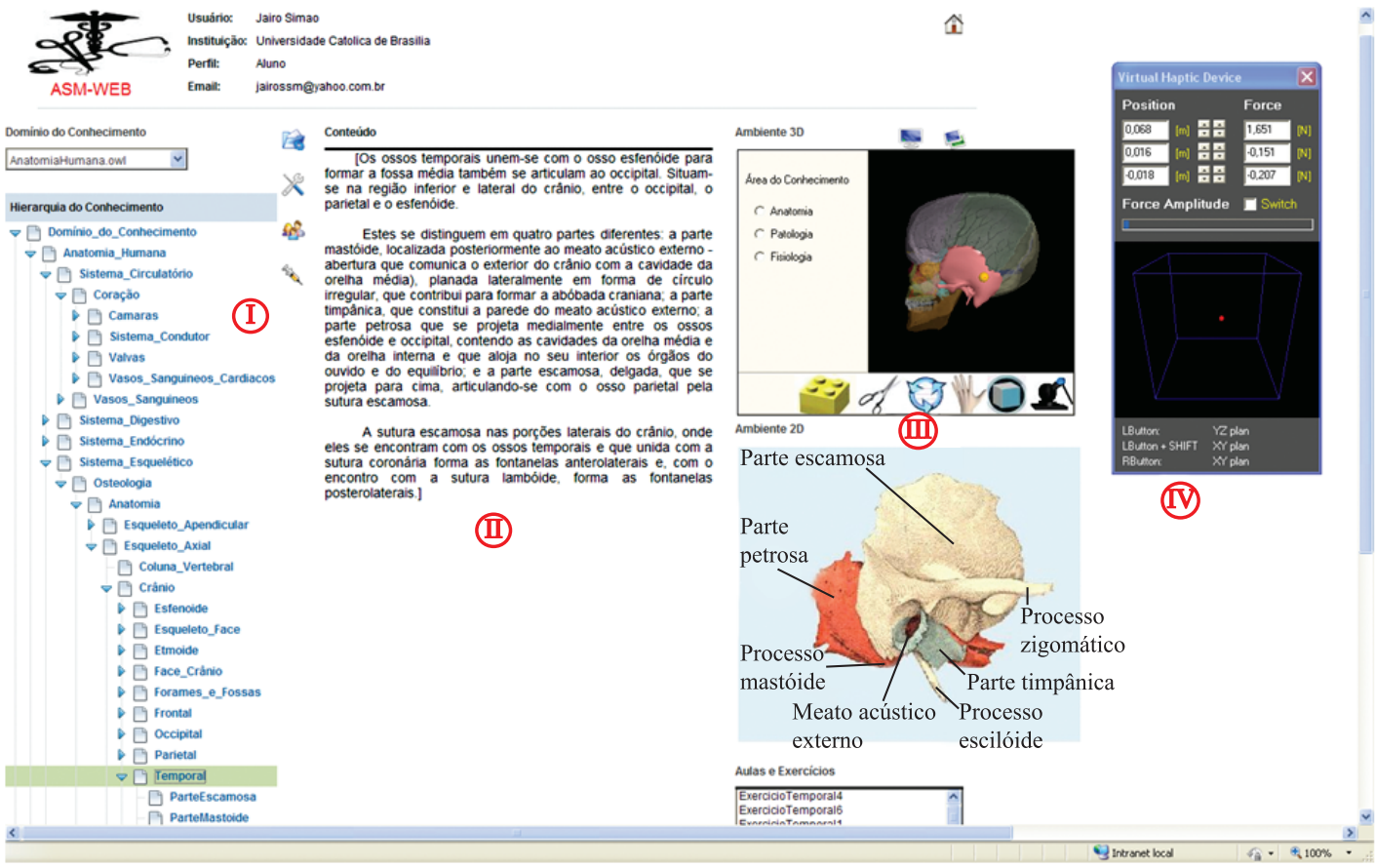

Figura 8. i) Interface do sistema ASM em anatomia óssea humana incluindo i) hierarquia do conteúdo ii) conteúdo textual, iii) visualização 3D em ambiente de RV, iv) controle e contato háptico.

Figure 8. i) System Interface ASM in human bone anatomy including i) hierarchy of content ii) textual content, iii) $3 D$ visualization in VR environment, iv) control and haptic contact.

que são limitados a uma descrição textual, imagens bidimensionais e aulas de anatomia em laboratórios especializados, além do alto custo, muitas vezes gerando conflitos éticos. Nesse sentido, o ASM vem contribuir com um processo adicional de ensino da anatomia humana, disponibilizando uma ferramenta 3D para visualização, navegação, interação, conteúdo formalizado por especialista e integrado a simulação em ambiente web, bem como está disponível a todos que realizem um pré-cadastro.

Dentro desse escopo, específico dessa arquitetura, buscou-se definir requisitos de avaliação detalhados na Tabela 3 e expostos sumariamente a seguir (Melo, 2007):

- Design gráfico;

- Conteúdo textual;

- Facilidade, simplicidade e disponibilidade de acesso ao sistema via internet;

- Visualização e navegação 3D;

- Inter-relacionamento entre os diferentes conceitos da anatomia (navegação vertical) e com os conceitos da fisiologia e patologia (navegação horizontal);

- Interação com a estrutura 3D a partir de interfaces hápticas;

- Facilidade de navegação bidirecional (STI Û RV).

\section{Resultados}

Em função da experimentação, alguns problemas operacionais foram detectados e solucionados. Estes problemas referiam-se i) à estabilidade do sistema em um ambiente multiusuários com diferentes tipos de sistemas operacionais, ii) capacidade de gerenciamento do servidor remoto, iii) armazenamento e recuperação de arquivos, e iv) localização e visualização das estruturas 3D.

Apesar da limitação do ponto de vista de significância estatística, os resultados animadores indicaram algo mais que indícios e eventuais tendências. Em relação à tecnologia e à arquitetura empregadas, observou-se que os propósitos almejados neste experimento foram alcançados. Em relação ao grupo A:

- $86 \%$ foram favoráveis ao uso da tecnologia web para disseminação do conhecimento na área médica;

- $78 \%$ consideraram que RV contribuiu para um melhor entendimento das estruturas anatômicas humanas;

- $63 \%$ consideraram que a manipulação direta de objetos 3D contribuiu para um melhor entendimento das estruturas anatômicas; 
Tabela 2. Questionário de validação do sistema.

Table 2. Questionnaire for system validation.

\begin{tabular}{|c} 
Universidade Católica de Brasília - UCB \\
Mestrado em Gestão do Conhecimento e da Tecnologia da Informação - MGCTI \\
Campus II, SGAN 916, Módulo B, Sala A 207 \\
Asa Norte, 70790-160 Brasília - DF, Brasil
\end{tabular}

A pesquisa faz parte de uma dissertação que está sendo desenvolvida no MGCTI.

Questionário de Avaliação do Protótipo: Ambiente de Simulação Médica - ASM

\begin{tabular}{|ll|}
\hline \multicolumn{2}{|c|}{ Questionário de Avaliação do Protótipo: Ambiente de Simulação Médica - ASM } \\
\hline \multicolumn{2}{c|}{ Perfil } \\
\hline Curso: & Semestre: \\
\hline Instituição: & Titulação:
\end{tabular}

\begin{tabular}{|c|c|}
\hline \multicolumn{2}{|c|}{ Usabilidade } \\
\hline Como você classifica seu conhecimento em anatomia? & - Ruim • Razoável • Bom • Ótimo \\
\hline $\begin{array}{l}\text { Quando você acessou o sistema, o mesmo estava disponível, com } \\
\text { todas as funcionalidades? }\end{array}$ & • Sim • Não \\
\hline $\begin{array}{l}\text { O acesso ao sistema pela Internet facilita a disseminação do } \\
\text { conhecimento na área da saúde? }\end{array}$ & • Sim • Não \\
\hline A instalação do sistema foi difícil? & • Sim • Não \\
\hline O design (Aspecto Visual) do sistema está apropriado? & - Sim • Não \\
\hline
\end{tabular}

\begin{tabular}{|l|l|}
\hline $\begin{array}{l}\text { As funcionalidades do sistema facilitaram a interação com o } \\
\text { conteúdo? }\end{array}$ & $\bullet$ Sim ・ Não \\
\hline $\begin{array}{l}\text { O conteúdo está de acordo com o que é apresentado nas aulas de } \\
\text { anatomia? }\end{array}$ & $\bullet$ Sim ・Não \\
\hline
\end{tabular}

Realidade Virtual

\begin{tabular}{|l|l|}
\hline $\begin{array}{l}\text { A realidade virtual contribuiu para o aprendizado sobre estruturas } \\
\text { anatômicas? }\end{array}$ & $\bullet$ Sim ・ Não \\
\hline $\begin{array}{l}\text { Como você classifica a qualidade das estruturas anatômicas 3D } \\
\text { disponibilizadas? }\end{array}$ & $\bullet$ Ruim ・ Razoável ・ Bom ・ Ótimo \\
\hline A forma de interação e navegação 3D está apropriada? & $\cdot$ Sim ・Não \\
\hline $\begin{array}{l}\text { Você conhece dispositivos háptico (Interfaces de Contato), isto é, } \\
\text { braços cirúrgicos? }\end{array}$ & $\bullet$ Sim ・Não \\
\hline $\begin{array}{l}\text { Você gostaria de aprender sobre procedimentos cirúrgicos pela } \\
\text { Internet? }\end{array}$ & $\bullet$ Sim ・Não \\
\hline $\begin{array}{l}\text { Você acha que o uso da Realidade Virtual foi o diferencial neste } \\
\text { sistema de ensino? }\end{array}$ & $\bullet$ Sim ・Não \\
\hline
\end{tabular}
sistema de ensino?

\section{Sistema para Ensino}

\begin{tabular}{|l|l|}
\hline $\begin{array}{l}\text { De que forma o sistema atendeu suas expectativas em relação a uma } \\
\text { ferramenta de apoio ao ensino de anatomia? }\end{array}$ & $\bullet$ Ruim ・ Razoável ・ Bom ・ Ótimo \\
\hline Que classificação você daria para o sistema? & $\bullet$ Ruim ・ Razoável ・ Bom ・ Ótimo \\
\hline Outras Considerações/Sugestões? & \\
\hline
\end{tabular}

- $63 \%$ aprovaram a utilização de sistemas web no ensino de procedimentos médicos;

- $67 \%$ avaliaram como positiva a utilização de interfaces hápticas associadas à RV no ensino da anatomia.

Quanto à organização e qualidade do conteúdo apresentado, 67\% do grupo B consideraram o ASM uma ferramenta de apoio que melhorou o ensino presencial de anatomia.

Em outras palavras, em decorrência das análises das respostas dos grupos A e B, a proposta de integração de
RV e STI baseada em todos os requisitos determinados para este trabalho, apresentou-se viável. Na Figura 9, é possível observar um gráfico desta situação, onde o conjunto de respostas positivas foi sempre superior ao grupo de respostas negativas.

Após a realização da pesquisa, o ASM foi divulgado em várias listas de especialistas de várias universidades brasileiras, sendo, posteriormente, registradas 514 solicitações de cadastro, para cursos de fisioterapia, nutrição, biologia e psicologia, que mantiveram a avaliação positiva em relação ao ASM. 
Tabela 3. Objetivos específicos sobre cada pergunta do questionário Table 3. Specific goals on each question

\begin{tabular}{|c|c|c|}
\hline $\mathbf{N}^{\mathbf{o}}$ & Questionamento & Objetivos Específicos \\
\hline 1 & Curso? Semestre? Instituição? Titulação? & $\begin{array}{l}\text { As seguintes informações buscam identificar o perfil do aprendiz } \\
\text { ou especialista que está consultando o sistema, buscando } \\
\text { estabelecer uma relação entre o tipo de conteúdo consultado no } \\
\text { sistema e o que é ministrado de maneira convencional. }\end{array}$ \\
\hline 2 & $\begin{array}{l}\text { Como você classifica seu conhecimento em } \\
\text { anatomia? }\end{array}$ & $\begin{array}{l}\text { Este questionamento busca verificar o grau de conhecimento do } \\
\text { aprendiz ou especialista antes do contato com o sistema. }\end{array}$ \\
\hline 3 & $\begin{array}{l}\text { Quando você acessou o sistema, o mesmo estava } \\
\text { disponível, com todas as funcionalidades? }\end{array}$ & $\begin{array}{l}\text { Este questionamento busca verificar se o aprendiz ou especialista } \\
\text { conseguiu acessar o sistema e se este estava com todas as opções } \\
\text { habilitadas. }\end{array}$ \\
\hline 4 & $\begin{array}{l}\mathrm{O} \text { acesso ao sistema pela Internet facilita a } \\
\text { disseminação do conhecimento na área da saúde? }\end{array}$ & $\begin{array}{l}\text { Busca verificar se o fato do sistema estar disponível em ambiente } \\
\text { Web contribuiu de alguma forma para o acesso e a disseminação } \\
\text { do conhecimento, ou se este deveria estar disponível localmente, } \\
\text { isto é instalado de forma individual. }\end{array}$ \\
\hline 5 & A instalação do sistema foi difícil? & $\begin{array}{l}\text { Busca verificar se a forma encontrada para a instalação das } \\
\text { bibliotecas do ambiente virtual é de fácil entendimento ou se } \\
\text { precisa de melhoria. }\end{array}$ \\
\hline 6 & $\begin{array}{l}\text { O design (Aspecto Visual) do sistema está } \\
\text { apropriado? }\end{array}$ & $\begin{array}{l}\text { Buscar verificar se o layout, imagens, botões e fundos de tela } \\
\text { simplificaram o acesso à informação e contribuíram para a } \\
\text { ergonomia do sistema. }\end{array}$ \\
\hline 7 & $\begin{array}{l}\text { As funcionalidades do sistema facilitaram a } \\
\text { interação com o conteúdo? }\end{array}$ & $\begin{array}{l}\text { Busca verificar se a interface está disposta de maneira a } \\
\text { simplificar o entendimento, por exemplo, exercícios próximos ao } \\
\text { conteúdo apresentado, a RV próxima à realidade estática. }\end{array}$ \\
\hline 8 & $\begin{array}{l}\text { O conteúdo está de acordo com o que é } \\
\text { apresentado nas aulas de anatomia? }\end{array}$ & $\begin{array}{l}\text { Busca verificar se existe divergência em relação ao conteúdo } \\
\text { ministrado nas aulas de anatomia e o formalizado pelo } \\
\text { especialista. }\end{array}$ \\
\hline 9 & $\begin{array}{l}\text { A RV contribuiu para o aprendizado sobre } \\
\text { estruturas anatômicas? }\end{array}$ & $\begin{array}{l}\text { Busca verificar a eficiência do uso da RV na compreensão das } \\
\text { estruturas anatômicas 3D. }\end{array}$ \\
\hline 10 & $\begin{array}{l}\text { Como você classifica a qualidade das estruturas } \\
\text { anatômicas } 3 \mathrm{D} \text { disponibilizadas? }\end{array}$ & $\begin{array}{l}\text { Busca verificar se as estruturas captadas por tomografias e } \\
\text { ressonância magnéticas possibilitam a geração de modelos 3D } \\
\text { com boa qualidade de entendimento. }\end{array}$ \\
\hline 11 & $\begin{array}{l}\text { A forma de interação e navegação 3D está } \\
\text { apropriada? }\end{array}$ & $\begin{array}{l}\text { Busca verificar se a adoção do mouse e das ferramentas } \\
\text { desenvolvidas para o ASM, contribuíram para o entendimento/ } \\
\text { interação das estruturas anatômicas 3D. }\end{array}$ \\
\hline 12 & $\begin{array}{l}\text { Você conhece dispositivos hápticos (Interfaces de } \\
\text { Contato), isto é, braços cirúrgicos? }\end{array}$ & $\begin{array}{l}\text { Busca verificar se o aprendiz ou especialista já tiveram algum } \\
\text { contato com braços robóticos. }\end{array}$ \\
\hline 13 & $\begin{array}{l}\text { Você gostaria de aprender sobre procedimentos } \\
\text { cirúrgicos pela Internet? }\end{array}$ & $\begin{array}{l}\text { Busca verificar se o aprendiz ou especialista tem interesse de } \\
\text { realizar treinamento de procedimentos médicos pela internet. }\end{array}$ \\
\hline 14 & $\begin{array}{l}\text { Você acha que o uso da RV foi o diferencial neste } \\
\text { sistema de ensino? }\end{array}$ & $\begin{array}{l}\text { Busca verificar se os requisitos propostos e identificados foram } \\
\text { atendidos da melhor forma possível. }\end{array}$ \\
\hline 15 & $\begin{array}{l}\text { De que forma o sistema atendeu suas expectativas } \\
\text { em relação a uma ferramenta de apoio ao ensino } \\
\text { de anatomia? }\end{array}$ & $\begin{array}{l}\text { Busca verificar se alguma forma o ASM-WEB-TUTOR } \\
\text { contribuiu para o aprimoramento do conhecimento do aprendiz } \\
\text { sobre anatomia humana. }\end{array}$ \\
\hline 16 & Que classificação você daria para o sistema? & Busca analisar de maneira geral a classificação do sistema. \\
\hline 17 & Outras Considerações/Sugestões? & Busca registrar as considerações \\
\hline
\end{tabular}

A partir destas novas avaliações foi possível traçar alguns pontos de melhoria do protótipo ASM, isto é:

- Tanto o grupo A quanto o grupo B consideraram uma solução de boa qualidade para o ensino da anatomia humana. Entretanto, o design web do ASM precisa ainda ser reavaliado.

- O uso de interfaces hápticas em ambientes de simulação como este, precisa ser mais bem difundido e aprimorado, pois esta tecnologia não é convencional no meio da saúde.
- O ASM deve se tornar, no futuro, um real objeto de ensino, baseando-se, por exemplo, em análise de perfil, segundo a interação com o ambiente.

\section{Discussão e Conclusão}

A arquitetura do sistema ASM proposta neste trabalho mostrou-se interessante por contribuir para as áreas de engenharia biomédica, informática na educação 

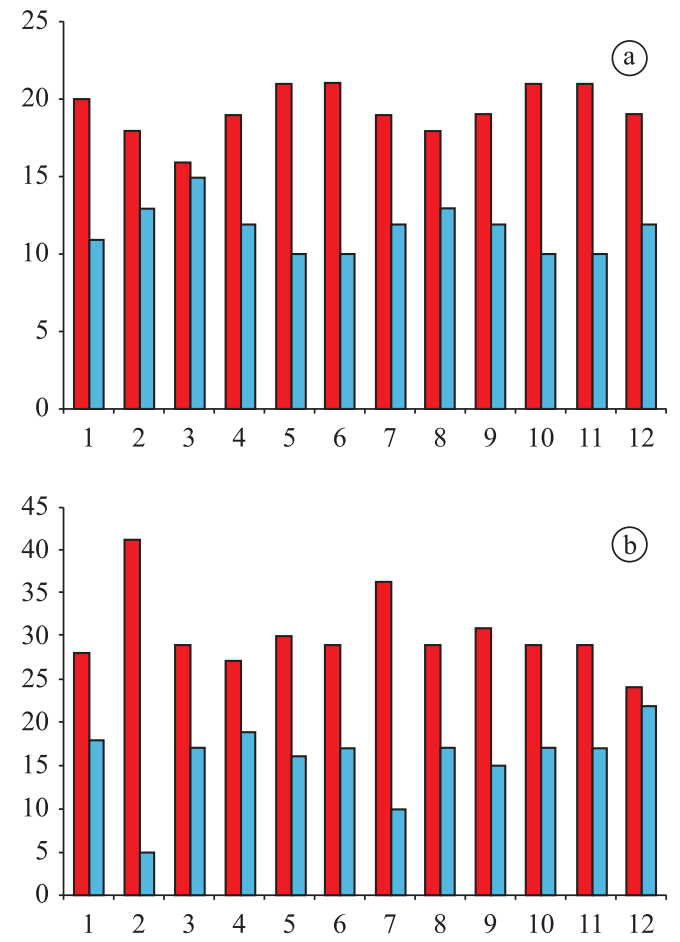

$\square \operatorname{Sim} \square$ Não

Figura 9. Gráficos comparativo das respostas dos grupos A e B. Figure 9. Comparative graphs for the answers of groups $A$ and $B$.

e educação em saúde, por propor um STI que i) formaliza seu conteúdo na forma de ontologias, ii) possibilita o relacionamento entre diferentes domínios do conhecimento em saúde (anatomia, fisiologia e patologia) através de uma navegação horizontal e vertical no conteúdo, iii) associa RV e ontologias, iv) possibilita uma visualização de estruturas 3D da anatomia humana em ambiente web, v) viabiliza a manipulação de estruturas anatômicas modeladas em um ambiente $3 \mathrm{D}$ via interfaces hápticas, e vi) proporciona uma navegação bidirecional entre o STI e a RV.

Como trabalhos futuros, são necessárias ainda algumas melhorias no ASM, isto é:

- Utilizar os conceitos da lógica fuzzy (Brasil, 2008; Zadeh, 1965) como funções fuzzy e graus de pertinências para as variáveis linguísticas, para se obter melhor qualidade das respostas quanto ao aprendizado do estudante e utilidade do ASM.

- Não foi foco desse trabalho avaliar o aprendizado do estudante quanto à aplicação do uso de estratégias pedagógicas. Uma possível melhoria na validação e atualização da arquitetura do ASM pode ser almejada, através da incorporação de uma rede neural artificial entre a interação do estudante e o processo de busca de informações na ontologia. Dessa forma, de acordo com a avaliação de características da interação e das avaliações realizadas pelo estudante durante a execução do sistema, o mesmo poderá ser direcionado ao tipo de conteúdo que melhor atenda à sua necessidade (Bittencourt, 2006).

- O escopo de atuação do sistema consistiu no processo de avaliação de apenas um dispositivo de interação, isto é, Phantom. Este equipamento disponibilizado pela Sensable não é acompanhado de documentação técnica de desenvolvimento, o que dificultou o processo de integração. Além disso, o processo de comunicação da interface háptica é baseado em memória compartilhada, sendo que algumas linguagens como Java ainda não disponibilizam interfaces para acessar nativamente esta estrutura em memória, sendo necessário criar as chamadas Java Native Interface (JNI), solicitando o recurso do sistema operacional. Esta estrutura em memória é criada/atualizada no momento em que a interface é ativada, sendo necessário sincronizar as configurações do ambiente de simulação com o status atual da interface háptica. Seria interessante abordar $o$ assunto de gerenciamento de interfaces hápticas, que ainda é um tema novo na área científica e merece uma discussão mais elaborada.

A arquitetura aqui apresentada não atende, por exemplo, a implementação em tempo real de processos de deformação de superfície para estruturas deformáveis da anatomia humana, como é o caso da musculatura mamária. Outro ponto será a reestruturação da camada WebService, que é responsável pelo encapsulamento do processamento da ontologia, buscando maior aderência à Arquitetura Orientada a Serviço (AOS).

\section{Agradecimentos}

À FINEP e à FINATEC/UNB/FAP-DF - que patrocinaram financeiramente o projeto do ano de 2006 a 2008 e 2009 , respectivamente.

\section{Referências}

Bittencourt ISP. Plataforma para construção de ambientes interativos de aprendizagem baseados em agentes [mestrado]. Maceió: Universidade Federal de Alagoas; 2006. 133 p.

Bezivin J, Lemesle R. Ontology-based layered semantics for precise OA\&D modeling. Lecture Notes in Computer Science. 1998; 1357:287-292,. 
Bowman DA, Kruijff E, Laviola J, Poupyrev I. An introduction to 3-D user interface design. Presence: Teleoperators and Virtual Environments. 2001; 10(1):96-108. http://dx.doi.org/10.1162/105474601750182342

Brasil LM. Informática em Saúde. Taguatinga: Universa; Londrina: Eduel; 2008.

Conti F. Environment Chai3D. [Internet]. 2010 jan [cited 2010 jan]. Available from: http://www.chai3d.org.

Dameron OR, Rubin DL, Musen M. Challenges in converting frame-based ontology into OWL: the foundational model of anatomy case-study. In: American Medical Informatics Association Annual Symposium: Proceedings of the American Medical Informatics Association Annual Symposium; 2005 oct; Washington, USA. Washington; 2005. p. 181-185.

Faeth A., Oren M., Harding C. Combining 3-D geovisualization with force feedback driven user interaction. In: ACM SIGSPATIAL: Proceedings of the $16^{\text {th }}$ ACM SIGSPATIAL International Conference on Advances in Geographic Information Systems; 2008 nov 5-7; Irvine, USA. New York: ACM; 2008. http://dx.doi.org/10.1145/1463434.1463466

Haarslev V, Möller R, Wessel M. Querying the semantic Web with Racer + nRQL. In: International Workshop on Applications of Description Logics (ADL'04): Proceedings of the KI-04 International Workshop on Applications of Description Logics; 2004 sep 24; Ulm, Germany. Ulm; 2004.

Liu CL, Uang ST. Measurement and prediction of cybersickness on older users caused by a virtual environment. In: International Conference UAHCI'07: Proceedings of the $4^{\text {th }}$ International Conference on Universal Access in
Human-Computer Interaction: Ambient Interaction; 2007; Berlin, Germany. Berlin: Springer-Verlag; 2007. p. 666-75.

Melo JSS. Integrando realidade virtual em sistemas tutores inteligentes no domínio da saúde [dissertação]. Brasília: Universidade Católica de Brasília; 2007. 115 p.

Moresi E. Manual de metodologia da pesquisa. Brasília: Universidade Católica de Brasília; 2003. 108 p.

Orgun B, Vu J. HL7 ontology and mobile agents for interoperability in heterogeneous medical information systems. Computers in Biology and Medicine. 2006; 36(7-8):817-36. PMid:16139263. http://dx.doi.org/10.1016/j.compbiomed.2005.04.010

Rönkkö J, Markkanen J, Launonen R, Ferrino M, Gaia E, Basso V, Patel H, D'cruz M, Laukkanen S. Multimodal astronaut virtual training prototype. International Journal of Human-Computer Studies. 2006; 64(3):182-91.

Russell, S.; Norvig, P. Inteligência artificial. Rio de Janeiro: Elsevier; 2004.

Santos BS, Dias P, Pimentel A, Baggerman JW, Ferreira C, Silva S, Madeira J. Head-mounted display versus desktop for 3D navigation in virtual reality: a user study. Multimedia Tools and Applications. 2009; 41(1):161-81. http://dx.doi. org/10.1007/s11042-008-0223-2

Wilson, JR, D'cruz M. Virtual and interactive environments for work of the future. International Journal of HumanComputer Studies. 2006; 64(3):158-69. International Conference

Zadeh LA. Fuzzy sets. Information and Control. 1965; 8:338-53. http://dx.doi.org/10.1016/S0019-9958(65)90241-X

\footnotetext{
Autores

Jairo Simão Santana Melo

Departamento de Engenharia Elétrica, Faculdade de Medicina, Universidade de Brasília - UnB, CEP 70910-900, Brasília, DF, Brasil

\section{Lourdes Mattos Brasil}

Programa de Pós-Graduação em Engenharia Biomédica, Faculdade Gama - FGA, Universidade de Brasília - UnB, CEP 70910-900, Brasília, DF, Brasil
}

\section{Carlos Eduardo Balbuena Panerai}

Faculdade de Fisioterapia, Universidade Católica de Brasília - UCB, QS 07 Lote 01 EPCT, Águas Claras, CEP 71966-700, Taguatinga, DF, Brasil

\section{Ana Paula Bernardi da Silva}

Centro de Ciência e Tecnologia - CCT, Universidade Católica de Brasília - UCB

QS 07 Lote 01 EPCT, Águas Claras, CEP 71966-700, Taguatinga, DF, Brasil 